\title{
Role of Low Dose Sildenafil in Improvement of Implantation Rate in Case of Recurrent ICSI failure
}

\author{
Yahia A. Wafa, Mohammed Moh. ALKhouly, Reham M. Salah Abdelsalam Mosalam \\ Department of Obstetrics and Gynecology, Al-Azhar University, Cairo, Egypt \\ Corresponding author: Reham M. Salah, Mobile: 00201150011938, Email: dr_rehamsalah@yahoo.com
}

\begin{abstract}
Background: Infertile couples to have healthy children are one of primary tasks of assisted reproductive technologies. In order to fulfill these tasks, reproductive medicine constantly needs to obtain information on physiology and pathophysiology of infertility and to develop efficient strategies for ovarian hyper-stimulation.

Objectives: Aim of the current Work was to evaluate the efficacy of oral sildenafil citrate $25 \mathrm{mg}$ on pregnancy outcome, endometrial thickness, pattern and uterine arteries Doppler blood flow in patients with 3 or more concoctive unexplained recurrent IVF/ICSI implantation failure.

Patients and Methods: This prospective, randomized, double blinded study included a total of seventy patients undergoing ICSI trial attending at the University Maternity Hospital IVFICSI unit. The included subjects were randomly allocated into two groups: Group I (study group): included 35 women with recurrent ICSI implantation failure with another trial of ICSI using the standard drugs and technique with the addition of sildenafil $25 \mathrm{mg}$ orally twice daily. Group II (control group): included 35 women with recurrent ICSI implantation failure with another trial of ICSI using the standard drugs and technique (control group).

Results: The statistical comparison between group I (Sildenafil Citrate) and group II (control) regarding endometrial thickness showed non significant differences before and significant differences after 21days of administration of Sildenafil Citrate. The statistical comparison in patients in group I (Sildenafil Citrate) regarding Pulsatility index, Resistance index and Systolic/ diastolic ratio showed significant differences of these parameters. Conclusion: It could be concluded from this study that adding of sildenafil citrate $25 \mathrm{mg}$ orally twice daily for 21 days to patients with unexplained recurrent ICSI implantation failure, increase in pregnancy rate.

Keywords: Sildenafil, Implantation Rate, Recurrent ICSI failure.
\end{abstract}

\section{INTRODUCTION}

Assisted reproductive techniques (ART) are used widely to treat fertility problems, which affect approximately $7-15 \%$ of women in reproductive age ${ }^{(\mathbf{1})}$. Although there have been several improvements in techniques during the last three decades, clinical pregnancy and live-birth rates remain at approximately $30-40 \%$ and $20-30 \%$, respectively ${ }^{(2)}$ according to results from the Canadian ART Register in 2007. Increasing these rates is desirable for couples undergoing ART, as treatment failure is a source of psychological distress and the most common cause of drop-out before achieving pregnancy ${ }^{(3)}$.

The success of in vitro fertilization and embryo transfer (IVF-ET) cycles depends mainly on embryo quality (4), uterine receptivity, and efficient crosstalk between the embryo and the receptive endometrium. It is thought that the impairment of any one of these factors or biological processes may result in implantation failure ${ }^{(5)}$.

Implantation remains a major limiting step of assisted reproductive technology (ART) and the uterine receptivity is important for successful implantation ${ }^{(6)}$.

Endometrial receptivity is regulated by many factors including endometrial perfusion ${ }^{(7)}$. Several studies have demonstrated the existence of a correlation between endometrial characteristics and pregnancy rate in IVF/ICSI patients ${ }^{(8)}$.

Factors affecting the growth of endometrium are still not well understood, but recently many researchers have focused on the angiogenesis and vascularization within the endometrium and found that a poor uterine receptivity in women with thin endometrium may be due to the impairment of blood flow impedance through the endometrium ${ }^{(\boldsymbol{9})}$.

Several treatment options were suggested to improve endometrial receptivity in IVF cycles. Using the available treatment modalities (extended estrogen administration, vaginal sildenafil, vitamin $\mathrm{E}$, pentoxifylline, and luteal phase GnRH-a supplementation) ${ }^{(\mathbf{1 0})}$.

It has been reported that low-dose aspirin is effective in improving implantation and pregnancy rates in the IVF program. We also reported that a nitric oxide donor reduced the uterine arterial PI of a woman with a history of repeated unexplained ICSI/IVF implantation failure. The transvaginal color Doppler technique makes it possible to further the investigation on the influence of various medications on uterine perfusion ${ }^{(\mathbf{1 1})}$.

Sildenafil citrate enhances the effect of NO by inhibiting phosphodiesterase type 5(PDE5) which is responsible for degradation of c GMP, So with the use of sildenafil cGMP remain elevated, which leads to vascular relaxation and increased blood flow to improve the endometrial thickness ${ }^{(\mathbf{1 2})}$.

Uterine receptivity is likely to be regulated by a number of factors including uterine perfusion and is of great importance in achieving a normal pregnancy. Transvaginal pulsed Doppler ultrasonography allows non-invasive evaluation of uterine circulation. In a number of studies, pulsed Doppler ultrasonography 
demonstrated blood flow changes in the uterus and ovaries during the menstrual cycle ${ }^{(13)}$.

The interest to assess endometrial vascularity has been on track in early studies since 1988. During in vitro fertilization, embryo transfer (IVF-ET) cycles, up to two-thirds of implantation failures are estimated to be caused by defects in endometrial receptivity ${ }^{\left({ }^{(14)}\right.}$. Efforts have been made to evaluate uterine, endometrial and sub endometrial blood supplies as a mirror of endometrial receptivity, especially during intrauterine insemination and IVF-ET cycles ${ }^{(\mathbf{1 5})}$.

The objective of this study was to evaluate the efficacy of low dose oral sildenafil citrate $25 \mathrm{mg}$ in pregnancy rate, endometrial thickness and uterine arteries Doppler flow indices before embryo transfer in predicting the success of IVF/ICSI cycles in patients with history of recurrent unexplained ICSI/IVFimplantation failure.

\section{PATIENTS AND METHODS}

This prospective, randomized, double blinded study included a total of seventy patients undergoing ICSI trial attending at the University Maternity Hospital IVFICSI unit. Verbal consent about the experimental nature of treatment prior to initiation of therapy and written informed consent from all the subjects were obtained. Approval of the Research and Ethics Committee of University Maternity Hospital, Cairo, Egypt in Accordance with local research governance requirements was obtained. The protocol was registered at clinical trials.gov. The study was done during the period from March 2016 to March 2018.

The included subjects were randomly allocated into two groups:

Group I (study group): included 35 women with recurrent ICSI implantation failure with another trial of ICSI using the standard drugs and technique with the addition of sildenafil $25 \mathrm{mg}$ orally twice daily.

Group II (control group): included 35 women with recurrent ICSI implantation failure with another trial of ICSI using the standard drugs and technique (control group).

All women included in the study had systematic investigations according to the established protocol including hysterosalpingogram, coagulation profile, thyroid function test, pelvic ultrasound scan, anti-cardiolipin antibodies $\operatorname{IgG}$ and $\operatorname{IgM}$, lupus anticoagulant antibodies, and hormonal analysis (including progesterone, prolactin, androgens, and early follicular FSH, and LH).

Inclusion criteria: The selected patients fulfilled the following criteria:

1- Age 18-35 years.

2- Repeated ICSI failure (women who have a prior history of recurrent ICSI failure which is defined as $>=3$ previous trials).

3- Infertile patients undergoing IVF/ICSI due to male factor, PCO, tubal factor or unexplained infertility.

4- Normal size and shape of the uterus as evidenced by transvaginal ultrasound or hesterography.

5- Infertile women whether it is primary or secondary infertility.

6- Regular menstrual pattern.

7- 3 or more months elapsed since the last ICSI trial.

8- Ability to attend follow up as planned.

9- Informed consent.

\section{Exclusion criteria:}

Patients having concomitant use of organic nitrites, or nitrate, severe hepatic, renal, or cardiovascular impairment, patients with history of stroke or myocardial infarction and patients with retinitis pigmentosa were excluded from the study.

\section{All women were subjected to}

1- Detailed past, family, and obstetric histories.

2- Ultrasonography and pulsed Doppler blood flow assessments, using a Medison (Sonoace X6, South Korea) scanner with a transvaginal multifrequency probe, was performed, to measure:

a- Maximum endometrial thickness (calculated as the maximum distance between each myometrial/endometrial interface through the central longitudinal axis of the uterus and with the calipers place don't eh outer walls of the endometrium).

b- Uterine arteries blood flow studies including:

i. Systolic/ diastolic ration which reflects intrinsic resistance of the uterine artery.

ii. Pulsatility index (calculated as the difference between the peak systolic and end-diastolic velocities divided by the mean velocity during the cardiac cycle) which is a measure of the variability of blood velocity in the uterine artery.

iii. Resistance index (calculated as the difference between the peak systolic and end-diastolic velocities divided by the peak systolic velocity) which is an indicator of the resistance of uterine artery to perfusion.

\section{Technique of ICSI:}

All patients underwent stimulation using long down regulation protocol starting from $21^{\text {st }}$ day of previous cycle. Human menopausal gonadotropin (HMG) was given starting from $3^{\text {rd }}$ day of cycle.

- Vaginal uls folliculometry was done starting from the $7^{\text {th }}$ day of the cycle then every other day.

- Human chorionic gonadotropin (HCG) was given in a dose with 10000 IU IM when at least 3 follicles reached $18-20 \mathrm{~mm}$.

- Retrieval of oocyte was done after 36h from HCG.

- Retrieved oocytes were injected under inverted microscope and fertilized by ICSI was done $48 \mathrm{~h}$ later. 


\section{Follow Up and assessment}

After informed consent, all the patients included had received an oral dose of $25 \mathrm{mg}$ of Sildenafil citrate as three time daily to day $21^{\text {st }}$ of same cycle after receiving the standard dose or induction of ovulation.

Transvaginal UIS: to detect endometrial thickness, number of follicles and uterine artery blood flow Doppler.

Outcome measures:

- Primary outcome: Pregnancy rate per cycle.

- Secondary outcome:

1) Endometrial thickness

2) Uterine artery blood flow Doppler

Sample size justification:

Sample size was calculated using EpiInfo® version 6.0 , setting the type- 1 error $(\alpha)$ at 0.05 and the power $(1-\beta)$ at 0.80 . Data from a previous study ${ }^{(13)}$, showed that the endometrial thickness was significantly higher in the sildenafil citrate group $(\mathrm{p}<0.0001)$, the triple line patterns of the endometrium were significantly higher in the sildenafil citrate group $(p<0.0001)$. The echogenic patterns of the endometrium were significantly higher in control group $(\mathrm{p}<0.0001)$. Finally, implantation rate and the clinical pregnancy rates were higher in the sildenafil citrate group. Calculation according to these values to find such a difference produced a minimal sample size of 67 cases. Assuming a drop-out ratio of 5\%, the sample size was 35 women in each group.

\section{Statistical analysis}

Data were analyzed using MedCalc $(\odot$ version 18.2.1 (MedCalc@ Software bvba, Ostend, Belgium). Continuous numerical variables were presented as mean \pm SD and inter-group differences were compared using the unpaired t test. The Welch test was used in place of the $t$ test whenever the assumption of normality was not fulfilled. Skewed variables were presented as median and interquartile range and differences were compared using the Mann-Whitney test.

Categorical variables were presented as number and percentage and differences were compared using the Pearson chi-squared test or the chisquared test for trend (for ordinal data).

Receiver-operating characteristic (ROC) curve analysis was used to examine the value of endometrial thickness or UA Doppler indices for prediction of clinical pregnancy. DeLong method was used to compare the areas under ROC curves (AUC). The AUC is interpreted as follows:

\section{RESULTS}

Table 1. Characteristics of both study groups

\begin{tabular}{l|ccccc}
\hline Variable & Sildenafil group $(\mathbf{n}=35)$ & Control group $(\mathbf{n}=35)$ & $\mathbf{t} / \boldsymbol{\chi}^{\mathbf{2}}$ & $\mathbf{d f}$ & $\mathbf{p}$-value* \\
\hline Age $(\mathrm{yr})$ & $32 \pm 4$ & $31 \pm 4$ & 0.833 & 68 & 0.408 \\
\hline Weight $(\mathrm{kg})$ & $69.2 \pm 13.4$ & $65.5 \pm 12.3$ & 1.179 & 68 & 0.242 \\
\hline Height $(\mathrm{cm})$ & $155 \pm 5$ & $156 \pm 7$ & -0.979 & 68 & 0.331 \\
\hline BMI $\left(\mathrm{kg} / \mathrm{m}^{2}\right)$ & $29.0 \pm 5.7$ & $26.9 \pm 4.7$ & 1.640 & 68 & 0.106 \\
\hline Infertility duration $(\mathrm{yr})$ & $6 \pm 2$ & $4 \pm 2$ & 2.781 & 68 & $\mathbf{0 . 0 0 7}$ \\
\hline Infertility type & & & 1.472 & 1 & $0.225 \#$ \\
\hline Iry & $23(65.7 \%)$ & $18(51.4 \%)$ & & \\
\hline 2ry & $12(34.3 \%)$ & $17(48.6 \%)$ & & & \\
\hline Number of previous ICSI & $4 \pm 2$ & $4 \pm 1$ & 0.687 & 68 & 0.494 \\
\hline
\end{tabular}

Data are mean $\pm \mathrm{SD}$ or number $(\%), \mathrm{t}=\mathrm{t}$ statistic; $\chi 2=$ chi-squared statistic; $\mathrm{df}=$ degrees of freedom, $*$ Unpaired $\mathrm{t}$ test unless otherwise specified, \#Pearson chi-squared test.

There was no significant difference between the two groups in age $(\mathrm{p}=0.408)$, weight $(\mathrm{p}=0.242)$, height $(\mathrm{p}=0.331)$ and $\operatorname{BMI}(\mathrm{p}=0.106)$. There were no significant difference between the two groups regarding type of infertility. All patients experienced failure more than 3 ICSI cycles. The two groups were suffering infertility for period between 2-10 years. The sildenafil group had significant longer duration of infertility $(\mathrm{p}=0.007)$ and more failed ICSI cycles.

Table 2. Results of hormonal assay in both study groups

\begin{tabular}{l|lllll}
\hline Variable & Sildenafil group $(\mathrm{n}=35)$ & Control group $(\mathrm{n}=35)$ & $\mathrm{t}$ & $\mathrm{df}$ & $\mathrm{p}$-value* \\
\hline B FSH $(\mathrm{mIU} / \mathrm{ml})$ & $6.84 \pm 1.56$ & $7.00 \pm 1.86$ & -0.396 & 68 & 0.693 \\
B LH $(\mathrm{mIU} / \mathrm{ml})$ & $6.50 \pm 1.72$ & $7.15 \pm 1.29$ & -1.084 & 68 & 0.282 \\
B E2 $(\mathrm{pg} / \mathrm{ml})$ & $37.97 \pm 2.22$ & $39.51 \pm 5.31$ & -0.466 & 68 & 0.643 \\
B AMH $(\mathrm{ng} / \mathrm{ml})$ & $3.00 \pm 1.51$ & $3.25 \pm 1.02$ & -0.594 & 68 & 0.554 \\
\hline
\end{tabular}

Data are mean \pm SD or number $(\%), t=t$ statistic; $d f=$ degrees of freedom., *Unpaired $t$ test.

The hormonal profile of the two groups showed no significant differences. 
Table 3. Endometrial thickness in both study groups

\begin{tabular}{|c|c|c|c|c|c|c|}
\hline Variable & Time & $\begin{array}{l}\text { Sildenafil group } \\
(n=35)\end{array}$ & $\begin{array}{l}\text { Control group } \\
(n=35)\end{array}$ & $\mathrm{t}$ & df & p-value* \\
\hline \multirow{3}{*}{$\begin{array}{l}\text { Endometrial thickness } \\
\qquad(\mathrm{mm})\end{array}$} & Day 6 & $8.60 \pm 1.05$ & $6.25 \pm 1.00$ & 9.584 & 68 & $<0.001$ \\
\hline & Day 8 & $9.91 \pm 1.25$ & $7.31 \pm 0.87$ & 10.100 & 68 & $<0.001$ \\
\hline & Day 10 & $11.06 \pm 1.59$ & $7.95 \pm 0.83$ & 10.305 & 51.332 & $<0.001$ \\
\hline
\end{tabular}

Data are mean $\pm \mathrm{SD}, \mathrm{t}=\mathrm{t}$ statistic; $\mathrm{df}=$ degrees of freedom, *Unpaired $\mathrm{t}$ test.

During $\mathrm{COH}$, the endometrium showed progressive significant thickening in the two studied groups between day 6 and day 10 of the cycle. However, the endometrium in the sildenafil group was significantly thicker from day 6 to day $10(\mathrm{p}=0.001)$.

Table 4. Endometrial pattern in both study groups

\begin{tabular}{|c|c|c|c|c|c|c|c|}
\hline Variable & Time & Pattern & $\begin{array}{l}\text { Sildenafil } \\
\text { group }(n=35)\end{array}$ & $\begin{array}{l}\text { Control group } \\
(n=35)\end{array}$ & $\chi^{2}$ & df & $\begin{array}{l}\text { p- } \\
\text { value* }\end{array}$ \\
\hline \multirow{3}{*}{$\begin{array}{l}\text { Endometrial } \\
\text { pattern }\end{array}$} & Day 6 & $\begin{array}{l}\text { Trilaminar } \\
\text { Echogenic }\end{array}$ & $\begin{array}{l}33(94.3 \%) \\
2(5.7 \%)\end{array}$ & $\begin{array}{l}26(74.3 \%) \\
9(25.7 \%)\end{array}$ & 5.285 & 1 & 0.022 \\
\hline & Day 8 & $\begin{array}{l}\text { Trilaminar } \\
\text { Echogenic }\end{array}$ & $\begin{array}{l}33(94.3 \%) \\
2(5.7 \%)\end{array}$ & $\begin{array}{l}26(74.3 \%) \\
9(25.7 \%)\end{array}$ & 5.285 & 1 & 0.022 \\
\hline & Day 10 & $\begin{array}{l}\text { Trilaminar } \\
\text { Echogenic }\end{array}$ & $\begin{array}{l}33(94.3 \%) \\
2(5.7 \%)\end{array}$ & $\begin{array}{l}26(74.3 \%) \\
9(25.7 \%)\end{array}$ & 5.285 & 1 & 0.022 \\
\hline
\end{tabular}

Data are number (\%).

$\chi^{2}=$ chi-squared statistic; $\mathrm{df}=$ degrees of freedom.

*Pearson Chi-squared test.

The endometrium showed trilaminar appearance in the most of sildenafil group, 33 out of 35 patients (94.3\%), and in 26 out of 35 patients of control group (74.5\%) starting from day 6 up to day 10 of the induced cycle $(p=0.002)$.

Table 5. UA Doppler indices in both study groups

\begin{tabular}{|c|c|c|c|c|c|c|c|c|}
\hline & & $\begin{array}{l}\begin{array}{l}\text { Sildenafil } \\
(\mathrm{n}=35)\end{array} \\
\end{array}$ & group & $\begin{array}{l}\begin{array}{l}\text { Control } \\
(\mathrm{n}=35)\end{array} \\
\end{array}$ & group & & & \\
\hline Variable & Time & Mean & SD & Mean & SD & $\mathrm{t}$ & $\mathrm{df}$ & p-value* \\
\hline \multirow{3}{*}{ UA PI } & Day 6 & 2.31 & 0.41 & 3.92 & 0.01 & -8.695 & 44.922 & $<0.001$ \\
\hline & Day 8 & 2.02 & 0.41 & 3.31 & 0.77 & -8.769 & 52.297 & $<0.001$ \\
\hline & Day 10 & 1.85 & 0.46 & 2.79 & 0.49 & -8.299 & 68 & $<0.001$ \\
\hline \multirow{3}{*}{ UA RI } & Day 6 & 0.65 & 0.19 & 0.90 & 0.06 & -7.405 & 39.934 & $<0.001$ \\
\hline & Day 8 & 0.56 & 0.18 & 0.85 & 0.06 & -8.873 & 41.659 & $<0.001$ \\
\hline & Day 10 & 0.52 & 0.19 & 0.86 & 0.06 & -10.119 & 41.024 & $<0.001$ \\
\hline \multirow{3}{*}{ UA S/D ratio } & Day 6 & 4.70 & 1.79 & 10.58 & 3.21 & -9.472 & 53.295 & $<0.001$ \\
\hline & Day 8 & 3.84 & 1.73 & 9.62 & 3.11 & -9.602 & 53.178 & $<0.001$ \\
\hline & Day 10 & 2.76 & 1.60 & 9.05 & 3.07 & -10.749 & 51.118 & $<0.001$ \\
\hline
\end{tabular}

Data are mean \pm SD.

$\mathrm{t}=\mathrm{t}$ statistic; $\mathrm{df}=$ degrees of freedom.

*Unpaired t test.

Using Doppler ultrasonography, the pulsitility index (PI), resistant index (RI) and systolic/diastolic ratio (S/D) of the uterine arteries showed progressive significant decrease in the two studied groups between day 6 and day 10 of the cycle. However, uterine arteries Doppler indices (PI, RI and S/D ratio) in sildenafil group was significantly lower from day 6 up to day $10(\mathrm{p}=0.001)$. 
Table 6. Pregnancy rate in both study groups

\begin{tabular}{llllll}
\hline Variable & & $\begin{array}{l}\text { Sildenafil } \\
(\mathbf{n = 3 5})\end{array}$ & group & Control group $(\mathbf{n = 3 5})$ & p-value* \\
\hline \multirow{2}{*}{ Chemical pregnancy } & Negative & $17(48.6 \%)$ & $27(77.1 \%)$ & \multirow{2}{*}{$\mathbf{0 . 0 2 5}$} \\
\hline \multirow{2}{*}{ Clinical pregnancy } & Positive & $18(51.4 \%)$ & $8(22.9 \%)$ & \multirow{2}{*}{$\mathbf{0 . 0 1 2}$} \\
\hline \multirow{2}{*}{ Number of gestational } & Negative & $17(48.6 \%)$ & $28(80.0 \%)$ & \\
sacs & Positive & $18(51.4 \%)$ & $7(20.0 \%)$ & \multirow{2}{*}{$\mathbf{0 . 0 0 3 \#}$} \\
& Nil sac & $17(48.6 \%)$ & $28(80.0 \%)$ & \\
\hline
\end{tabular}

Data are number and percentage $(\%)$.

*Fisher's exact test.

Shown that the majority of sildenafil group achieved clinical pregnancy 18 out of 35 patients $51.4 \%$ compared to control group who achieved clinical pregnancy 7 out of 35 represents $20.0 \%, 9$ out of 35 patients in the control group $(\mathrm{p}=0.012)$.

This table shows that the majority of sildenafil group achieved pregnancy 18 out of 35 patents $51.4 \%$ compared to $20.0 \%, 7$ out of 35 patients in the control group ( $\mathrm{p}=0.012$ ) Consequence to increasing pregnancy outcome in sildenafil group, Table5 showed significant increasing in number of intrauterine gestational sacs in sildenafil group comparable to control group $(\mathrm{p}=0.002)$

Table 7. Implantation rate in both study groups

\begin{tabular}{l|lllll}
\hline Variable & Sildenafil group $(\mathrm{n}=35)$ & Control group $(\mathrm{n}=35)$ & Difference & Z-statistic & $\mathrm{p}$-value* \\
\hline Implantation rate $(\%)$ & $23.3+-26.6$ & $8.1+-16.4$ & 15.3 & 4.6 to 25.9 & $\mathbf{0 . 0 0 6}$ \\
\hline
\end{tabular}

Data are mean \pm standard deviation (SD).

$95 \% \mathrm{CI}=95 \%$ confidence interval.

*Unpaired t test.

Similarly, the implantation rate (no of sacs/ no of transferred embryos), was also significantly higher among sildenafil group ( 22 sacs per 94 transferred embryos) $23.40 \%$, than among control group ( 7 sacs per 89 transferred embryos) $8.1 \%$ (0.006).

Table 8. Relation between clinical pregnancy and endometrial thickness or UA Doppler indices

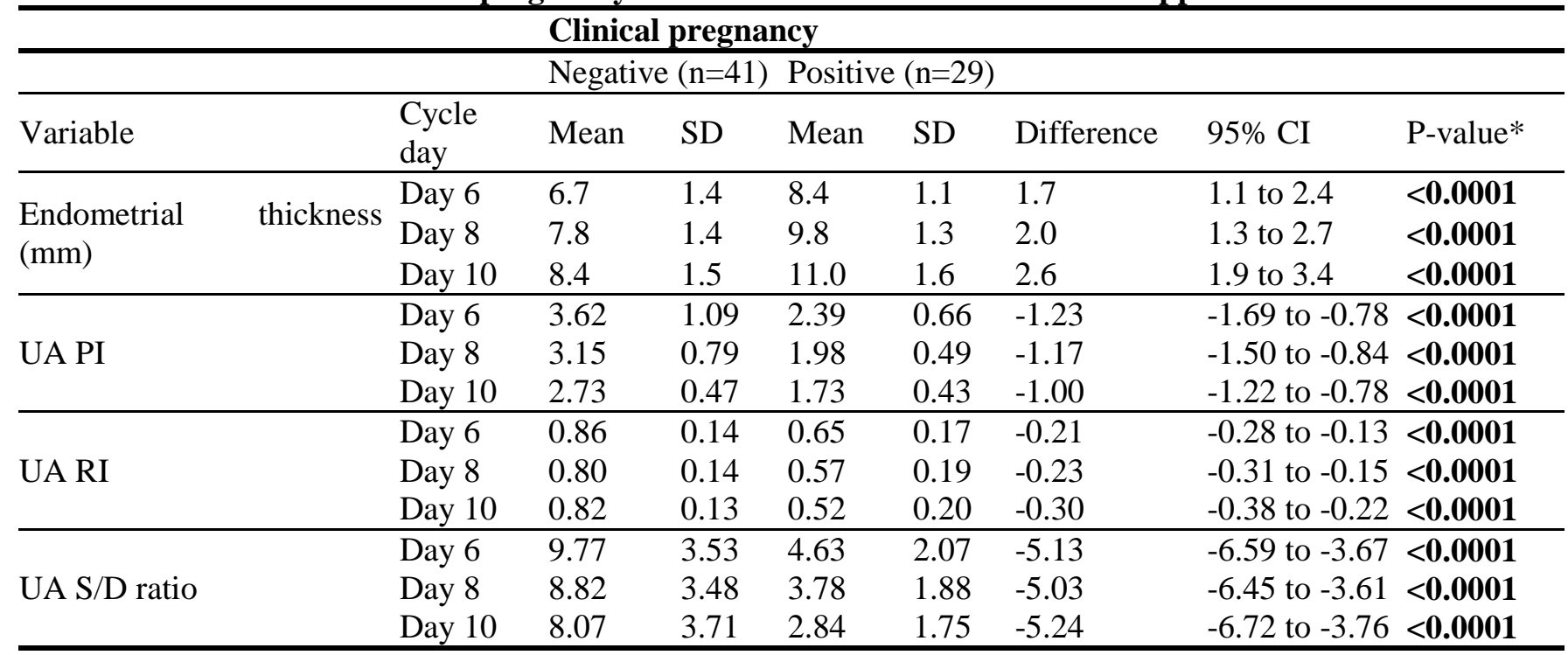

Data are mean and SD, SD $=$ standard deviation, $95 \% \mathrm{CI}=95 \%$ confidence interval.

*Unpaired t test.

Showed that in those patients who achieved pregnancy, have significant progressive increase in endometrial thickness and decrease in uterine artery Doppler indices than who did not achieved pregnancy $(\mathrm{p}=0.001)$. The endometrial thickness correlated significantly and negatively with Doppler indices. 
Table 9. Receiver-operating characteristic (ROC) curve analysis for the value of endometrial thickness or UA Doppler indices at day 10 in prediction of clinical pregnancy

\begin{tabular}{|c|c|c|c|c|}
\hline & \multicolumn{4}{|c|}{ Parameter on day 10} \\
\hline ROC parameter & ET & PI & RI & $\mathrm{SD}$ \\
\hline Area under the ROC curve (AUC) & 0.90 & 0.90 & 0.92 & 0.90 \\
\hline Standard Error (SE) & 0.04 & 0.04 & 0.03 & 0.04 \\
\hline 95\% Confidence interval (95\% CI) & 0.80 to 0.96 & 0.80 to 0.96 & 0.83 to 0.97 & 0.81 to 0.96 \\
\hline z statistic & 11.162 & 9.865 & 13.626 & 11.276 \\
\hline Significance level P $(\text { Area }=0.5)^{*}$ & $<0.0001$ & $<0.0001$ & $<0.0001$ & $<0.0001$ \\
\hline Youden index $\mathrm{J}$ & 0.69 & 0.70 & 0.66 & 0.63 \\
\hline Cut-off criterion & $>8.91$ & $<1.96$ & $<0.48$ & $<4.89$ \\
\hline Sensitivity $(\%)$ & 96 & $\overline{7} 2$ & 68 & 92 \\
\hline Specificity (\%) & 73 & 98 & 98 & 71 \\
\hline
\end{tabular}

*DeLong method.

The ROC analysis of endometrial thickness and uterine arteries Doppler indices at day 10 in relation to pregnancy outcome shoed significant p-value and large AUC which means that, based on the endometrial thickness and uterine arteries Doppler at day 10 of the cycle, it is possible to predict the possibility of positive pregnancy outcome in this ICSI cycle.

Further analysis using ROC was done to detect the best cutoff value pf endometrial thichness and uterine arteries Doppler indices at which the possibility of positive pregnancy test can be predicted.

Sensitivity represents the ability of the test to exclude positive cases.

Specificity represents the ability of the test to exclude negative cases.

\section{DISCUSSION}

Our results showed non statistical significant difference ( $p>0.05)$ regarding past history as age $(\mathrm{p}=0.408)$, weight $(\mathrm{p}=0.242)$, body mass index $(\mathrm{p}=0.106)$ between group I (sildenafil citrate) and group II (control) before drug administration. Also there was no statistical significant difference regarding laboratory investigations, also size of right and left ovaries were comparable in the two groups. Our results are in accordance with Hale et al. ${ }^{(16)}$ who found no significant differences in any measured physiologic characteristics between control and sildenafil treatment groups.

There were no significant differences between women of both groups regarding duration, type, etiology of infertility and duration of $\mathrm{COH}(\mathrm{p}=0.338)$. The number of retrieved oocyte showed significantly increased in sildenafil group (0.001). There were no significant difference regarding number, grade, and day of embryo transfer $(0.122)$. These results were in agreement with the results obtained by Zollner $\boldsymbol{e t} \boldsymbol{a l}$. (17) who measured uterine artery blood flow in the preimplantation period in embryo transfer cycles before embryo transfer in 233 patients. They found no differences in terms of age, duration of infertility, body mass index, cause of infertility, quality and number of transferred embryos between both groups.

There were no significant differences between women of both groups regarding finding of HSG, laparoscopy and hysteroscopy. These results were in agreement with the results obtained by Gaber ${ }^{(18)}$ who evaluate the role of ultrasound power Doppler in prediction of endometrial receptivity in 40 patients undergoing ICSI and found no differences in terms of HSG, laparoscopy and hysteroscopy, FHS, LH and TSH between both groups.

Endometrium pattern, endometrium thickness, and end-diastolic blood flow were shown to be the most effective combination for evaluation of uterine receptivity ${ }^{(16)}$. While Wang et al. ${ }^{\left({ }^{(19)}\right.}$ suggested that uterine artery S/D, RI and PI could not be used alone to predict endometrial receptivity.

In this study, the endometrium showed trilaminar appearance in the most of sildenafil group 33 out of 35 patients $94.3 \%$ and 26 out of 35 patients of control group $74.5 \%$ start from day 6 to day 10 of the induced cycle $(\mathrm{p}=0.054)$.

As regard endometrial thickness and pattern $\mathbf{A l}$ Mohammady et al. ${ }^{(20)}$ studied 100 infertile women Endometrial thickness and pattern were recorded on the day of HCG administration Clinical pregnancy rate (CPR) was calculated they resulted that trilaminar pattern showed the highest incidence of pregnancy Analyzing CPR with different endometrial patterns and endometrial thickness group revealed that trilaminar pattern with an endometrial thickness of $10-12.9 \mathrm{~mm}$ gives significantly higher CPR, they concluded that Endometrial thickness of 10-12.9 mm with trilaminar pattern is associated with higher CPR with ICSI cycles.

The use of the vasodilator drug, Sildenafil citrate to increase the uterine and endometrial blood flow has been previously reported with different results. In 2000, Sher and Fisch ${ }^{(21)}$ reported four patients who previously had an endometrial thickness 
between 5-7 $\mathrm{mm}$ in a prior failed IVF or IUI cycles, and who were all given vaginal sildenafil $25 \mathrm{mg}$ four times per day for 8-12 days during $\mathrm{COH}$. The endometrial thickness measured on day of hCG administration and was found to be between 8 and 12 $\mathrm{mm}$.

Our results agree with Dehghani et al. ${ }^{(22)}$ study, they found that the endometrial thickness was significantly higher in the sildenafil citrate group, the triple line patterns of the endometrium were significantly higher in the sildenafil citrate group.

In our study, during controlled ovarian hyper stimulation $(\mathrm{COH})$, the endometrium showed progressive significant thickening in the two studied groups between day 6 and day 10 of the cycle. However, the endometrium in the Sildenafil group was significantly thicker from day 6 to day 10 .The study showed that there was a significant statistical difference in endometrial thickness between treatment and control group which was higher in the treatment group, this might be attributed to the vasodilator effect of sildenafil citrate which lead to increase uterine blood flow ${ }^{(31)}$. This is in agreement with the studies done by Jerzak et al. ${ }^{(23)}$ who reported that there was a good correlation between endometrial thickness and the prevalence of conception. An endometrial thickness of $>9 \mathrm{~mm}$ in the late proliferative phase as determined by vaginal ultrasound, correlates well with the chance of pregnancy.

Also in agree with our study, a hospital based prospective study included 100 women with primary or secondary infertility randomized to receive sildenafil citrate $25 \mathrm{mg}$ vaginally every 6 hours for 5 days from day 8 of the cycle or estradiol valerate $2 \mathrm{mg}$ 6-8 hourly. At the time of hCG trigger, the mean endometrial thickness increased in the two groups, while endometrial vascularity was significantly better in sildenafil group. The clinical pregnancy rates were $20 \%$ in sildenafil group and $14 \%$ in estradiol group after 3 cycles of IUI ${ }^{\text {(24). }}$.

Parallelly to our study, the results from study done Paulus et al. ${ }^{(25)}$ found that sildenafil scientifically increase endometrial thickness to $>7 \mathrm{~mm}$ from day 8 to day 13 of the last menstrual period through a prospective study was done on twenty eight women by administration of $25 \mathrm{mg}$ (half of $50 \mathrm{mg}$ tablet) sildenafil citrate from day 8 of the cycle then reevaluation of those patients was done by transvaginal ultrasound on day 13 of the cycle for endometrial thickness and the number and sizes of (GF graafian follicles).

This results coincides with the results of Sher and Fisch (21), who evaluated the effect of vaginal sildenafil on the outcome of in vitro fertilization (IVF) after multiple IVF failures attributed to poor endometrial development in a cohort of 105 infertile women aged $<40$ years, with normal ovarian reserve and at least 2 consecutive prior IVF failures attributed to inadequate endometrial thickness. Patients were given vaginal sildenafil suppositories in addition to gonadotrophin releasing hormone $25 \mathrm{mg}, 4$ times daily, for 3-10 days. The results of the mentioned study showed that vaginal sildenafil enhanced endometrial development in $70 \%$ of studied cases.

In contrast to our results, Check et al. ${ }^{(26)}$ failed to demonstrate the beneficial effect of sildenafil on thin endometrium in women treated with estrogen and sildenafil citrate. Endometrial thickness was compared between the groups. Neither vaginal estrogen nor sildenafil significantly improved endometrial thickness or blood flow.

Merce ${ }^{(27)}$ postulated that endometrial blood flow reflects properly the uterine receptivity because the endometrium is the site where embryonic implantation takes place. Sildenafil act as an effective treatment for increasing uterine blood flow in patients at highest risk for sub-optimal uterine perfusion.

Clinical use of ultrasonic technology has increased as a way to track changes in the uterine wall thickness, in the assessment of the developmental potential of the basal layer of the endometrium ${ }^{(28)}$.

Wang et al. ${ }^{(19)}$ found that patients with detected uterine and endometrial blood flow had a higher pregnancy and implantation rate. Blood supply and distribution of the endometrium had a strong association with the possibility for embryo implantation and development, indicating the value of detecting the existence of uterine blood flow in predicting IVF-ET outcomes.

In our study, the resistance Index (RI) of the uterine artery showed progressive significant decrease in the Sildenafil group between day 6 and day 10 of the cycle. RI of the uterine arteries in the Sildenafil group was significantly lower compared to control group from day 6 to day 10 . The control group did not show significant change throughout the induced cycle.

Also, in agreement of our results, Hale et al. ${ }^{(1)}$, who studied the effect of sildenafil on uterine artery blood flow in non-pregnant nulliparous women. In this study, 15 women were randomized in a double-blind fashion to receive either placebo or sildenafil ( 25 or $100 \mathrm{mg}$ ) during the luteal phase of the menstrual cycle, color Doppler ultrasound of both uterine arteries was performed at baseline and 1 and 3-hours post-dosing to evaluate RI and uterine volumetric flow, the results showed that those who received sildenafil significantly decreased $\mathrm{RI}(\mathrm{p}=0.03)$.

Similar to our results, Malinova et al. ${ }^{(29)}$ had found significant decrease in PI and RI of uterine artery in patient group received $25 \mathrm{mg}$ sildenafil citrate.

In the present study, the systolic/diastolic ratio (SD) of the uterine artery showed progressive 
significant decrease in the two studied groups between day 6 and day 10 of the cycle. However, SD of the uterine arteries in the Sildenafil group were significantly lower from day 6 to day 10 .

Dzięciol et al. ${ }^{(30)}$ used color Doppler examination for analysis of the uterine wall perfusion. A significant decrease of values of PI and SV/DV ratio as well as an increase of end diastolic velocity and time averaged maximum velocity was noted. With the use of color coded sonography, the increased intensity of the blood flow in the uterine wall was observed with the use of sildanfil citrate.

The results of the current study goes with the results of Alatas and Yagci ${ }^{(31)}$, who studied the effect of single oral dose of $50 \mathrm{mg}$ sildenafil in 25 volunteer women which were subjected to color Doppler sonography before and 1 hour after administration of sildenafil. The study showed that RI was significantly lowered $(p<0.001)$ and pulsatility index also was significantly lowered $(\mathrm{p}<0.001)$.

The present study data showed that the majority of Sildenafil group achieved pregnancy compared to $40 \%$ of the control group $(\mathrm{p}=0.012)$.

Our study agree with Jerzak et al. ${ }^{(23)}$ in their study on Thirty-eight non-pregnant women with a history of recurrent ICSI/IVF implantation failure (RIF), and 37 healthy women with previous successful pregnancy outcomes Patients selfadministered sildenafil suppositories $(25 \mathrm{mg}$ intravaginal, four times a day) for 36 days. In the implantation failure women measuring uterine artery blood flow and endometrial thickness were recorded using Doppler ultrasound with an intravaginal probe. Endometrial thickness was significantly lower in the RIF group compared with the control group (8.06_2.74 mmvs. 9.03_3.34 $\mathrm{mm}$, respectively; $\mathrm{P}<.05)$. Endometrial thickness was significantly increased after vaginal sildenafil therapy compared with before therapy in the RIF group $\left(9.25 \_2.23 \mathrm{~mm}\right.$ vs.8.06 _ $2.74 \mathrm{~mm}$, respectively; $\mathrm{P}<.05$ ); however, we did not determine any significant differences in uterine artery PI before and after therapy (right uterine artery PI: $2.38 \_1.06$ vs. 2.26 _ 0.60 ; left uterine artery PI $2.30_{-} 0.69$ vs. $2.38 \_0.77$; respectively; $\mathrm{P}>.05)$. There were no significant differences in uterine artery PI between the RIF group and the control group (right uterine artery PI: $2.38 \ldots 1.06$ vs. 2.37 _ 0.36 ; left uterine artery PI $2.30 \_0.69$ vs. 2.40 _ $0.4 \overline{5}$; respectively; $\mathrm{P}>.05$ ).

Chen et al. ${ }^{(32)}$ studied a total of 2896 cycles all fresh IVF or ICSI treatment cycles that used the long protocol assessing endometrial thickness and pattern by 2-D vaginal sonographic assessment on the day of hCG administration they concluded that Combined analysis of endometrial thickness and pattern on the day of hCG administration was a better predictor of the outcome of IVF/ICSI-ET and may be more helpful for patient counseling than the separate analyses.

Singh et al. ${ }^{(33)}$ studied 101 infertile patients assessing endometrial thickness, pattern and uterine arteries blood flow by 2-D transvaginal power Doppler. They resulted that among the conceived

Women the endometrial thickness was $6-12 \mathrm{~mm}$, the endometrial echo pattern did not correlate with pregnancy outcome (have no prognostic value for pregnancy). The uterine blood flow directly correlated with the outcome of IVF cycles they concluded that with the thin endometrium $(\leq 7 \mathrm{~mm})$ with no triple line endometrial pattern coexisting in IVF/ICSI candidate's cryopreservation should be recommended. With a thin endometrium and good texture (triple line) other prognostic factors such as embryo quality should be taken into account.

Gao et al ${ }^{\text {(34) }}$ studied 119 infertile patients on the day of HCG injection assessing uterine and endometrial blood flow by Transvaginal color Doppler, endometrial pattern and thickness. They resulted that when the uterine and endometrial blood flow was high, the endometrial receptivity(ER) would be better, and the rate of pregnancy and the implantation would also increase obviously and concluded that uterine and endometrial blood flows measured by vaginal color Doppler ultrasound is a good predicator of pregnancy during IVF treatment. They think endometrial and sub endometrial blood flow examination is beneficial to assess ER. The value of uterine and endometrial blood flow can be used as a predictor of the final outcome of IVF/ICSI-ET.

The contradiction between previous published results can be explained by multiple methodological differences, especially in the time of the cycle when examinations take place and the Doppler parameters. Other factors could be invoked as well, including the differences in the study population and the stimulation, embryo transfer protocols ${ }^{(34)}$.

\section{CONCLUSION}

It could be concluded from this study that adding of sildenafil citrate $25 \mathrm{mg}$ orally twice daily for 21 days to patients with unexplained recurrent ICSI implantation failure, increase in pregnancy rate to be $(51.4 \%)$ than control group (20.0\%).

Based on these findings, it is possible to propose the use of transvaginal ultrasonography with Doppler flowmeter to assess uterine artery flow, local perfusion and obtain a prognostic cut-off value in women at risk of implantation failure in-vitro fertilization programs could also use this method to identify women with a higher probability for endometrial receptivity and adequate embryo development before the embryo is transferred.

We propose the use of transvaginal Doppler ultrasonography to detect impaired uterine perfusion 
as a screening test for women with RIF. Further studies on uterine arterial blood flow elucidated the mechanism of implantation failure associated with impaired uterine perfusion.

\section{REFERENCES}

1. Thoma ME, McLain AC, Louis JF et al. (2013): Prevalence of infertility in the United States as estimated by the current duration approach and a traditional constructed approach. Fertil Steril., 99: 1324-1331.

2. Sunkara SK, Rittenberg $\mathrm{V}$, Raine-Fenning $\mathbf{N}$ et al. (2011): Association between the number of eggs and live birth in IVF treatment: an analysis of 400135 treatment cycles. Hum Reprod., 26: 1768-1774.

3. Verberg MF, Eijkemans MJ, Heijnen EM et al. (2008): Why do couples drop-out from IVF treatment? A prospective cohort study. Hum Reprod., 23: 2050 2055.

4. Harper JC, Harton G (2010): The use of arrays in preimplantation genetic diagnosis and screening, Fertil Steril., 94:1173-1177.

5. Maruyama T (2009): Therapeutic Strategies for Implantation Failure due to Endometrial Dysfunction. J Mammal Ova Res., 26:129-133

6. Ledee N, Chaouat G, Serazin V et al. (2008): Endometrial vascularity by three-dimensional power Doppler ultrasound and cytokines: a complementary approach to assess uterine receptivity. J Reprod Immunol., 77:57-62.

7. Ng EH, Chan CC, Tang OS et al. (2006): The role of endometrial and subendometrial blood flows measured by three-dimensional power Doppler ultrasound in the prediction of pregnancy during IVF treatment. Hum. Reprod., 21: 164-170.

8. Richter KS, Bugge KR, Bromer JG et al. (2007): Relationship between endometrial thickness and embryo implantation, based on 1,294 cycles of in vitro fertilization with transfer of two blastocyst-stage embryos. Fertil Steril., 87:53-59.

9. Takasaki A, Tamura H, Miwa I et al. (2010): Endometrial growth and uterine blood flow: a pilot study for improving endometrial thickness in the patients with a thin endometrium. Fertil Steril., 93(6):1851-8.

10. Demir B, Dilbaz S, Cinar O et al. (2013): Estradiol supplementation in intracytoplasmic sperm injection cycles with thin endometrium. Gynecological Endocrinology: The Official Journal of the International Society of Gynecological Endocrinology, 29 (1), 42-5.

11. Mikiya N, Habara T, Soichi N et al. (2003): Impaired uterine arterial blood flow in pregnant women with recurrent pregnancy loss. J Ultrasound Med., 22: 2731.

12. Wareing M, Myers JE, O'Hara M et al. (2004): Effects of a phosphodiesterase-5 (PDE5) inhibitor on endothelium-dependent relaxation of myometrial small arteries. Am J Obstet Gynecol., 190:1283-1290.

13. Tan AL, Zaidi J and Campbell S (1996): Blood flow changes in the ovarian and uterine arteries during the normal menstrual cycle. Am J Obstet Gynecol., 175: 625-631.

14. Achache H, Revel A (2006): Endometrial receptivity markers, the journey to successful embryo implantation. Hum Reprod., 12(6):731-746.

15. Revel A (2012): Defective endometrial receptivity. Fertil Steril., 97(5):1028-32.

16. Hale SA, Jones CW, Osol G et al. (2010): Sildenafil Increases Uterine Blood Flow in Non-pregnant Nulliparous Women. Reproductive Sciences (Thousand Oaks, Calif), 17(4):358-365.

17. Zollner U, Specketer $M$ and Dietl J (2012): Uterine artery blood flow in the periimplantation period in embryo transfer cycles. Asian Pacific Journal of Reproduction, 1(3): 177-182

18. Gaber ME (2010): Evaluation of the Role of ThreeDimensional Ultrasound and Power Doppler in Prediction of Endometrial Receptivity in ICSI Patients. MSc Thesis, Department of Gynecology and Obstetrics. University of Ain Shams.

19. Wang L, Qiao J, Li R et al. (2010): Role of endometrial blood flow assessment with color Doppler energy in predicting pregnancy outcome of IVF-ET cycles: Reprod Biol Endocrinol., 108:122.

20. Al Mohammady M, Abdel Fattah G, Mahmoud M (2013): The impact of combined endometrial thickness and pattern on the success of intracytoplasmic sperm injection (ICSI) cycles. Middle East Fertility Society Journal, 18: 165-170, 2013.

21. Sher G, Fisch JD (2002): Effect of vaginal sildenafil on the outcome of in vitro fertilization (IVF) after multiple IVF failures attributed to poor endometrial development. Fertil Steril., 78(5):1073-6.

22. Dehghani R, Davar R, Hojjat F et al. (2013): Effect of sildenafil citrate on endometrial preparation and outcome of frozen-thawed embryo transfer cycles: a randomized clinical trial. Iranian Journal of Reproductive Medicine, 11(2):151-158.

23. Jerzak M, Monika K, Mrozek J et al. (2008): Sildenafil citrate decreased natural killer cell activity and enhanced chance of successful pregnancy in women with a history of recurrent miscarriage. Fertility and Sterility, 90 (5): 18481854.

24. Mangal S, Mehirishi S (2016): To study and compare the effect of vaginal sildenafil and estradiol valerate on endometrial thickness, blood flow and pregnancy rates in infertile women undergoing intrauterine insemination. Int $\mathrm{J}$ Reprod Contracept Obstet Gynecol., 5:2274-7

25. Paulus WE, Strehler E, Zhang M et al. (2002): Benefit of vaginal sildenafil citrate in assisted reproduction therapy. Fertility and Sterility, 77(4):8467.

26. Check JH, Graziano V, Lee G et al. (2004): Neither sildenafil nor vaginal estradiol improves endometrial thickness in women with thin endometria after taking oral estradiol in graduating dosages. Clin Exp Obstet Gynecol., 31:99-102. 
27. Merce LT (2002): Ultrasound markers of implantation. Ultrasound Rev Obstet Gynecol., 2:110123.

28. Pauls RN, Kleeman SD and Karram MM (2005): Female sexual dysfunction: Principles of diagnosis and therapy. Ostet Gynecol Surv., 60 (3): 196-205.

29. Malinova M, Abouyta $T$ and Krasteva $M$ (2013): The effect of vaginal sildenafil citrate on uterine blood flow and endometrium in the infertile women. Akush Ginekol (Sofiia), 52 (1):26-30.

30. Dzięcioł M, Stańczyk E, Noszczyk-Nowak A et al. (2014): The influence of Sildenafil citrate on uterine tissue perfusion and the cardiovascular system during the luteal phase of the ovarian cycle in cows. Acta Histochem., 116(2):377-81.

31. Alatas $\mathbf{E}$ and Yagci AB (2004). The effect of sildenafil citrate on uterine and clitoral arterial blood flow in postmenopausal women. Med Gen Med., 6(4): 51-56.

32. Chen S L, Wu F R, Luo C et al. (2010): Combined analysis of endometrial thickness and pattern in predicting outcome of in vitro fertilization and embryo transfer: a retrospective cohort study. Reprod Biol Endocrinol., 8:30-39.

33. Singh M, Chaudhry P, Asselin E (2011): Bridging endometrial receptivity and implantation: Network of hormones, cytokines, and growth factors. J Endocrinol., 210(1):5-14.

34. Gao M, Zhao Z, Li W et al. (2007): Assessment of Uterine Receptivity by Endometrial and Subendometrial Blood Flows Measured by Vaginal Color Doppler Ultrasound in Women Undergoing IVF Treatment. Journal of Reproduction \& Contraception, 18(3):205-212. 